\title{
Type 2C Phosphatase 1 of Artemisia annua L. Is a Negative Regulator of ABA Signaling
}

\author{
Fangyuan Zhang, Xueqing Fu, Zongyou Lv, Qian Shen, Tingxian Yan, Weiming Jiang, \\ Guofeng Wang, Xiaofen Sun, and Kexuan Tang
}

Plant Biotechnology Research Center, Fudan-SJTU-Nottingham Plant Biotechnology R\&D Center, School of Agriculture and Biology, Shanghai Jiao Tong University, 800 Dongchuan Road, Shanghai 200240, China

Correspondence should be addressed to Kexuan Tang; kxtang1@yahoo.com

Received 1 July 2014; Revised 27 August 2014; Accepted 29 August 2014; Published 28 October 2014

Academic Editor: Jose R. Botella

Copyright ( 2014 Fangyuan Zhang et al. This is an open access article distributed under the Creative Commons Attribution License, which permits unrestricted use, distribution, and reproduction in any medium, provided the original work is properly cited.

\begin{abstract}
The phytohormone abscisic acid (ABA) plays an important role in plant development and environmental stress response. Additionally, ABA also regulates secondary metabolism such as artemisinin in the medicinal plant Artemisia annua L. Although an earlier study showed that ABA receptor, AaPYL9, plays a positive role in ABA-induced artemisinin content improvement, many components in the ABA signaling pathway remain to be elucidated in Artemisia annua L. To get insight of the function of AaPYL9, we isolated and characterized an AaPYL9-interacting partner, AaPP2C1. The coding sequence of AaPP2C1 encodes a deduced protein of 464 amino acids, with all the features of plant type clade A PP2C. Transcriptional analysis showed that the expression level of AaPP2C1 is increased after ABA, salt, and drought treatments. Yeast two-hybrid and bimolecular fluorescence complementation assays (BiFC) showed that AaPYL9 interacted with AaPP2C1. The P89S, H116A substitution in AaPYL9 as well as G199D substitution or deletion of the third phosphorylation site-like motif in AaPP2C1 abolished this interaction. Furthermore, constitutive expression of $\mathrm{AaPP} 2 \mathrm{Cl}$ conferred $\mathrm{ABA}$ insensitivity compared with the wild type. In summary, our data reveals that $\mathrm{AaPP} 2 \mathrm{C} 1$ is an AaPYL9-interacting partner and involved in the negative modulation of the ABA signaling pathway in A. annua L.
\end{abstract}

\section{Introduction}

The phytohormone abscisic acid (ABA) is a key regulator of plant developmental processes and plant responses to abiotic stresses including drought, salt, osmotic, and cold stress $[1,2]$. ABA acts through a complex signaling cascade to induce changes in gene expression and in adaptive physiological responses [3]. In 2009, members of the PYR1/PYL/RCAR family of proteins (hereafter referred to as PYLs for simplicity) are proved to be ABA receptors in the cytoplasm and nucleus [4]. Upon ABA binding, the PYLs interact with and inhibit most members of the clade A subfamily of type$2 \mathrm{C}$ protein phosphatases (PP2Cs) [5]. ABA-mediated PP2C inhibition leads to the activation of subclass III SnRK2s [6]. Once activated, SnRK2s phosphorylate the downstream transcription factors and slow sustained (S-type) anion channels [7]. In this model, PP2C serves as a central and negatively regulated hub in $\mathrm{ABA}$ signaling [8] and the first connection of the ABA signaling with reversible phosphorylation.

Though plant PP2C is encoded by multigene family with 80 and 78 members in Arabidopsis and rice, respectively [9], only clade A subfamily is considered to be involved in ABA signaling [10]. Currently, at least six PP2Cs belonging to clade $\mathrm{A}$ are known to negatively regulate $\mathrm{ABA}$ signaling, namely, ABI1, ABI2, PP2CA/AHG3, AHG1, HAB1, and HAB2 [11]. Many of these protein phosphatases are induced by ABA. Two Arabidopsis strong ABA-insensitive loci, ABI1 and $\mathrm{ABI} 2$, have been demonstrated to encode PP2C enzymes, which functionally inhibit SnRK2s autophosphorylation [12]. Studies on the knockout and double mutants in ABIl and $\mathrm{ABI} 2$ confirmed that $\mathrm{ABI}$ and $\mathrm{ABI} 2$ have partially redundant function as inhibitor of ABA signaling [13].

In addition to Arabidopsis, many PP2Cs have been studied in various plant species [14]. In rice, three PP2Cs 
were demonstrated interaction with OsPYR/RCAR5 in ABA dependent manner [15]. When screening PP2Cs involved in beechnut dormancy, FsPP2C1 is identified and characterized as a negative regulator of ABA signaling in seed dormancy [16]. Subsequently, FsPP2C1 is proved interaction with Arabidopsis ABA receptor PYL8 in nucleus [17]. Overexpression of $P P 2 C$ gene from maize decreased plant tolerance to drought and salt in Arabidopsis [18]. In moss Physcomitrella patens, genetic evidence demonstrated that two PP2Cs functionally acted as a negative regulator in ABA signaling, which indicate that ABA signaling is evolutionarily conserved between Arabidopsis and P. patens [19]. In addition to negative regulator in $\mathrm{ABA}$ responses, a $\mathrm{PP} 2 \mathrm{C}$ from strawberry was reported to be a negative regulator in fruit ripening process [14].

Malaria is a global health problem especially in torrid zone, with more than one billion people living in areas with a high risk of the disease [20]. Artemisinin, isolated from traditional Chinese herb Artemisia annua L. (Qing $\mathrm{Hao}$ ), is a sesquiterpene lactone endoperoxide that provides the basis for effective treatments of malaria especially for the cerebral and the chloroquine-resistant forms of this disease [21]. Besides the antimalarial activity, artemisinin has also been reported to antiviral [22], anticancer [23], and antischistosomal activities [24]. We previously reported that the content of artemisinin is induced by exogenous ABA [25] and overexpression of AaPYL9, a functional ABA receptor in Artemisia [26].

To get deeper insight into the ABA signaling in Artemisia and ABA-regulated secondary metabolism, we started a search for putative $\mathrm{PP} 2 \mathrm{C}$ which interacts with ABA receptor AaPYL9. A cDNA library was constructed from Artemisia leaves and sequenced. Bioinformatics analysis identified that a PP2C belongs to clade A PP2C subfamily, which is named AaPP2C1. AaPP2C1 interacts with AaPYL9 in yeast two-hybrid and BiFC assays. Moreover, overexpression of AaPP2C1 in Arabidopsis produces insensitivity to ABA in seed germination and root elongation. Taken together, our data reveal that AaPP2C1 is an AaPYL9-interaction partner and play a negative regulator role in $\mathrm{ABA}$ signaling.

\section{Materials and Methods}

2.1. Chemicals, Plant Materials, Growth Conditions, and Stress Treatments. Abscisic acid was obtained from Sigma-Aldrich (http://www.sigmaaldrich.com); Artemisia annua L. used in this study was the same as previously described which were grown in a controlled environment with 16/8-h light/dark photoperiod at $26^{\circ} \mathrm{C}$ [26]. For abiotic stresses and ABA treatment, 1-month-old A. annua were treated with $300 \mathrm{mM} \mathrm{NaCl}$ and $10 \mu \mathrm{MABA}$, followed by sampling at $0,3,6$, and $12 \mathrm{~h}$. Drought stress was performed by leaving the intact 1-monthold $A$. annua in the air without water supply, followed by sampling at $0,3,6$, and $12 \mathrm{~h}$.

The Arabidopsis (Arabidopsis thaliana) were grown in pots in a growth chamber under $24^{\circ} \mathrm{C}$ and 16-h-light/8-hdark photoperiod at 80 to $100 \mathrm{mE} \mathrm{m-2} \mathrm{s-2.} \mathrm{To} \mathrm{examine} \mathrm{the}$ expression of ABA-responsive genes, wild type and AaPP2C1overexpression plants grown in liquid medium were treated with $10 \mu \mathrm{M}$ ABA for $3 \mathrm{~h}$, and the expression levels of RD29A, $R D 29 B, P 5 C S 1$, and $R A B 18$ were determined. For statistical analysis, at least three independent experiments were performed. $P$ values were calculated using the Student's $t$-test.

2.2. Mutagenesis of AaPYL9. The point mutation of AaPYL9 used in this study was described previously [26]. The mutation of $A a P P 2 C 1$ was performed using overlapping PCR strategy as work in AaPYL9.

2.3. Plasmid Construction. To overexpression of AaPP $2 \mathrm{C} 1$ in Arabidopsis, the coding sequence of $\mathrm{AaPP} 2 \mathrm{Cl}$ was amplified using the following primers: AaPP2C1-F-Bgl: ( $5^{\prime}$-GAAGATCTATGGAAGATATCCCTCCTTC- $\left.3^{\prime}\right)$ and AaPP2C1-R-Sac: (5'-GCGTCGACTCAAGATTTAGTTTTAAACC- $3^{\prime}$ ). After being confirmed by sequencing, the fragment was cloned into the BamHI and SacI sites of binary vector $\mathrm{pHB}$ under the control of the double CaMV $35 \mathrm{~S}$ promoter.

For yeast two-hybrid assay, the full length CDS as well as series of deletion and point mutation of $\mathrm{AaPP} 2 \mathrm{Cl}$ were cloned into pGADT7 vector between BamHI and SalI sites with the following primers: AaPP2C1-F-Bgl and AaPP2C1R-Sal (5' -GCGTCGACTCAAGATTTAGTTTTAAACC-3'). The full length CDS and point mutation of AaPYL9 were amplified by the following primers: AaPYL9-F-EcoR ( $5^{\prime}$-CGGAATTCATGAAGTACAGTAAGAAAGT- $\left.3^{\prime}\right)$ and AaPYL9-R-BamH (5'-CGGGATCCTCACTGAGTAATGTTCAGC- $3^{\prime}$ ), the PCR fragments inserted into EcoRI and BamHI sites of pGBKT7 vector subsequently.

For subcellular localization of AaPP2C1, the CDS of AaPP2C1 was amplified by PCR and cloned into gateway cloning vector pENTR (Invitrogen), then subsequently transferred to the destination vector pEarleyGate104 [27] by Gateway LR recombination reaction (Invitrogen), thus generating pEarleyGate104-YFP-AaPP2C1 construct. The pEarleyGate104-YFP-AaPYL9 construct used in this study was described previously [26].

BiFC vectors pEarleyagte201-YN and pEarleygate202-YC were kindly provided by Professor Steven J. Rothstein [28]. pENTR-AaPYL9 was transferred into pEarleyagte202YC and pENTR-AaPP2C1 was transferred into pEarleyagte201YN by Gateway LR recombination reaction (Invitrogen), named pEG202YC-AaPYL9 and pEG201YN-AaPP2C1, respectively.

2.4. Construction of AaPP2C1 Transgenic Arabidopsis. The pHB-AaPP2C1 construct was introduced into Agrobacterium tumefaciens strain GV3101 and then infiltrated into Arabidopsis with floral-dip method. The transgenic seedlings were selected in hygromycin medium $\left(25 \mu \mathrm{g} \mathrm{mL}^{-1}\right)$. T2 plants that produced $100 \%$ hygromycin-resistant plants in $\mathrm{T} 3$ generation were considered homozygous and used for further studies.

2.5. Real-Time Quantitative PCR Analysis. The real-time quantitative PCR analysis was performed as previously described [26]. Briefly, total RNA was extracted from roots, 
stem, leaves, flower buds, flowers, and samples after treatment. The relative expression levels were normalized by the housekeeping gene ACTIN. To examine the expression of ABA-responsive genes, total RNA was isolated from wild type and AaPP2C1-overexpression Arabidopsis as described above. The primers used in this research were described in Supplementary Table 1 (see Supplementary Material available online at http://dx.doi.org/10.1155/2014/521794).

2.6. Yeast Two-Hybrid Assay. Sets of bait and prey constructs were cotransformed into AH109 yeast strain. The transformed yeast cells were selected on synthetic minimal double dropout medium deficient in Trp and Leu. Protein interaction tests were assessed on triple dropout medium deficient in Trp, Leu, and His or quadruple dropout medium deficient in Trp, Leu, His, and adenine. At least six clones were analyzed, and experiments were repeated three times with similar results.

2.7. Subcellular Localization and BiFC Analysis. The subcellular localization analysis was performed as previously described [26]. BiFC assays were conducted according to $\mathrm{Lu}$ et al. [28]. Briefly, the constructs of AaPP2C1-cYFP, AaPP2Clm1-cYFP, and AaPP2C1m2-cYFP were introduced into A. tumefaciens strain GV3101 as well as AaPYL1-nYFP, AaPYL9m1-nYFP, and AaPYL9m2-nYFP constructs. Pairs of combinations were coinfiltrated into $N$. benthamiana. YFP signal was observed 48 to $60 \mathrm{~h}$ after being infiltrated by confocal laser scanning microscope (Leica TCS SP5-II). Results were confirmed by at least three times repeats.

2.8. Germination and Root Growth Assay. The germination and root growth assay were performed as previously described [26]. Briefly, the wild type and transgenic Arabidopsis seeds were plated on MS medium with or without different concentrations of $\mathrm{ABA}(0.1 \mu \mathrm{M}$ and $0.3 \mu \mathrm{M})$ after being stratified for $3 \mathrm{~d}$ at $4^{\circ} \mathrm{C}$. Seedling establishment was scored as the percentage of seeds that developed green expanded cotyledons and the first pair of true leaves at $7 \mathrm{~d}$. Root growth was measured after $7 \mathrm{~d}$ of transferring of 5-dayold seedlings onto vertical MS plates containing $5 \mu \mathrm{M} \mathrm{ABA}$ for ABA sensitivity assay.

\section{Results and Discussion}

3.1. Isolation and Sequence Analysis of AaPP2C1 in A. annua. Recently, we cloned and characterized AaPYL9, an ABA receptor orthologue in $A$. annua, which plays an important role in regulating artemisinin biosynthesis [26]. To identify the partners and downstream targets of AaPYL9, we performed bioinformatic analysis of cDNA library by BLAST-P program. A candidate gene which encodes a PP2C protein and contains the typical PYLs interaction domain was selected for further study. The cDNA fragment of this gene was isolated from $A$. annua via RT-PCR and designated as $A a P P 2 C 1$ which was 1392 bp long and encodes 463 amino acids. The deduced AaPP2C1 protein had a calculated molecular mass of $50.2 \mathrm{kDa}$ and a predicted pI of 4.92 (http://web.expasy.org/compute_pi/).
The phylogenetic relationship between AaPP2C1 and 68 Arabidopsis PP2Cs was shown in Supplementary Figure 1. Phylogenetic analysis indicated that $\mathrm{AaPP} 2 \mathrm{Cl}$ is clustered with group A PP2Cs of Arabidopsis. Sequence alignment with ABI1, ABI2, HAB1, and HABI2 from Arabidopsis reveals that $\mathrm{AaPP} 2 \mathrm{Cl}$ had the same motif with them, such as PA(phosphatidic acid-) binding site, PYLs interaction site, and a conserved PP2C domain (Figure 1).

3.2. AaPP2C1 Mainly Expressed in Leaves and Is Induced by ABA Treatment and Abiotic Stress. Expression pattern analysis can provide important clues for biological function. Quantitative RT-PCR was used to examine the expression pattern of $A a P P 2 C 1$ gene in different tissue and under different treatments. As shown in Figure 2, the transcripts of AaPP2C1 could be detected in whole plants including root, stem, leaves, and flowers, while they highly accumulates in leaves; this is in accord with the expression pattern of AaPYL9 [26]. On account of many identified ABA signalling component genes were induced under stress condition and ABA treatment; the expression level of $A a P P 2 C 1$ under drought stress, $\mathrm{ABA}$, and $\mathrm{NaCl}$ solution treatment were determined by quantitative real-time PCR as well. The results showed that the expression of $A a P P 2 C 1$ was significantly induced under either drought stress or $\mathrm{ABA}$ treatment or $\mathrm{NaCl}$ treatment. The similar expression pattern in different tissue between AaPP2C1 and AaPYL9 suggests that AaPP2C1 might interact with AaPYL9. The inducible transcript of AaPP2C1 suggested that this gene participates in ABA or drought responses.

3.3. Subcellular Localization of AaPP2C1. To determine the subcellular localization of AaPP2C1 in plant cells, we performed in vivo targeting experiments in tobacco (Nicotiana benthamiana). Both 35S: YFP-AaPP2C1 and 35S: YFPAaPYL9 constructs were delivered into epidermis leaf cell of tobacco by $A$. tumefaciens infiltration. Consistent with subcellular localization in Arabidopsis protoplasts as previously reported [26], YFP-AaPYL9 fluorescence was observed in both the nucleus and the cytosol (Figure 3(a)). Similar to YFP-AaPYL9, AaPP2C1-YFP fluorescence signal was observed in nucleus and cytosol. In addition, ABA treatment did not modify the subcellular localization of AaPP2C1YFP and AaPYL9-YFP under our experimental conditions (Figure 3(a)).

3.4. AaPP2C1 Interact with AaPYL9 in Yeast Two-Hybrid Assay. We previously reported that AaPYL9 interacted with ABI1 but not ABI2, HAB1, or HAB2 from Arabidopsis in yeast two-hybrid assay and in planta. To test whether the AaPP2C could interact with AaPYL9, yeast two-hybrid assay was performed between AaPP2C1 and AaPYL9. The full length of AaPP2C1 was fused to the GAL4 activation domain (GAD) while AaPYL9 was fused to the GAL4 DNA binding domain (GBD), generating $\mathrm{AD}-\mathrm{AaPP} 2 \mathrm{Cl}$ and $\mathrm{BD}$ AaPYL9 constructs, respectively. Yeast cells cotransformed with $\mathrm{AD}-\mathrm{AaPP} 2 \mathrm{C} 1$ and $\mathrm{BD}-\mathrm{AaPYL} 9$ grew well on selective medium, whereas the combination of BD-AaPYL9 with empty pGADT7 did not activate transcription of the HIS3 


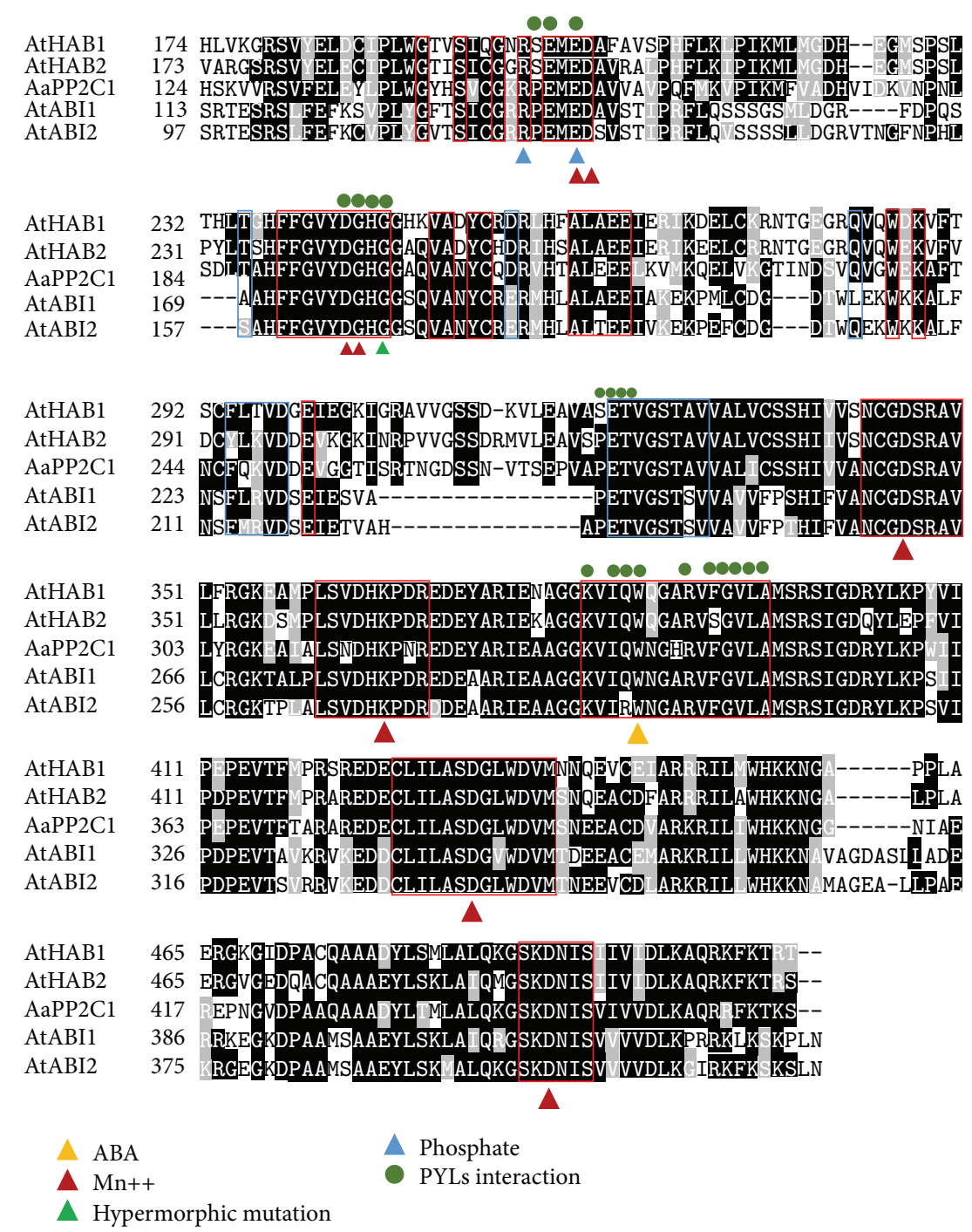

FIgure 1: Alignment of AaPP2C1 with four Arabidopsis PP2Cs. Amino acid sequence alignment of Artemisia AaPP2C1 with four Arabidopsis PP2Cs. Red frame indicates the amino acid residues involved in the interaction with ABA receptors; blue frame indicates the amino acid residues involved in the phosphorylation. The different icon indicates contact points with phosphate, metal, ABA, hypermorphic mutations and two mutation sites produced in this study.

and $A D E 2$ reporter gene (Figure 3(b)). This result indicated that AaPP2C1 could interact with AaPYL9 in yeast twohybrid assay.

Recent structural and genetic researches revealed that a gate-latch-lock mechanism in ABA receptors physically interacts with PP2Cs $[4,29]$. To address the question of whether AaPP2C1 might function in ABA signaling through physical interaction with AaPYL9 underlying the gate-latchlock mechanism, two mutated AaPYL9s were generated, AaPYL9m1 (substitution mutation P89S which is located on gate region) and AaPYL9m2 (substitution mutation H116A which is located on latch region), then subsequently fused to GBD. In combination with the prey construct AD-AaPP2C1, the GBD-AaPYL1m1 as well as GBD-AaPYL9m2 did not activate the reporter genes transcription (Figure 3(b)). These results showed that AaPP2C1 interact with AaPYL9 underlying gate-latch-lock mechanism, and the mutations on gate or latch region can disrupt the AaPP2C1-AaPYL9 interaction.

Eukaryotic PP2Cs containing a converse catalytic domain at either the $\mathrm{N}$ - or the C-terminus [30]. The metal coordinating centre in the catalytic domain is critical for their phosphatase activity and physiological function in ABA signaling. The dominant ABA-insensitive ABI1G180D (encoded by abi1-1) and ABI2G168D (encoded by abi2-1) disrupt the interaction between the $\mathrm{PP} 2 \mathrm{Cs}$ and the ABA receptors PYR/PYL/RCAR in Arabidopsis [4, 29]. In addition, a CDPK phosphorylation site-like motif (CPL) in ABI2 is required for the interactions of ABI2 with PYL5 and PYL9 [31]. To investigate the important site of $\mathrm{AaPP} 2 \mathrm{C} 1$ involved in interaction with AaPYL9, a point substitution mutation located on catalytic domain (AaPP2C1m1) and a deletion mutation (four amino acid residues from 305 to 308: RGKE) of $\mathrm{AaPP} 2 \mathrm{C1}$ (AaPP2C1m2) were generated and subsequently 


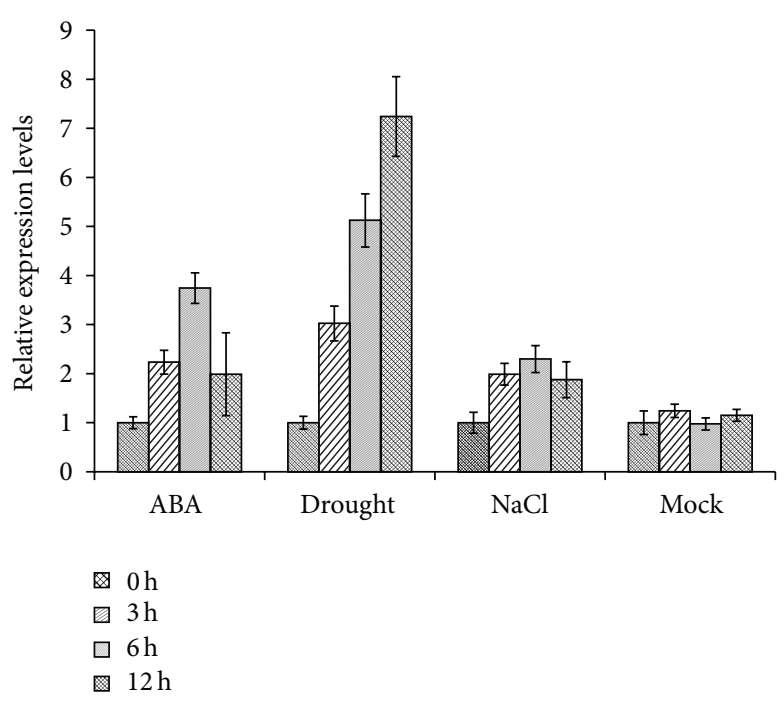

(a)

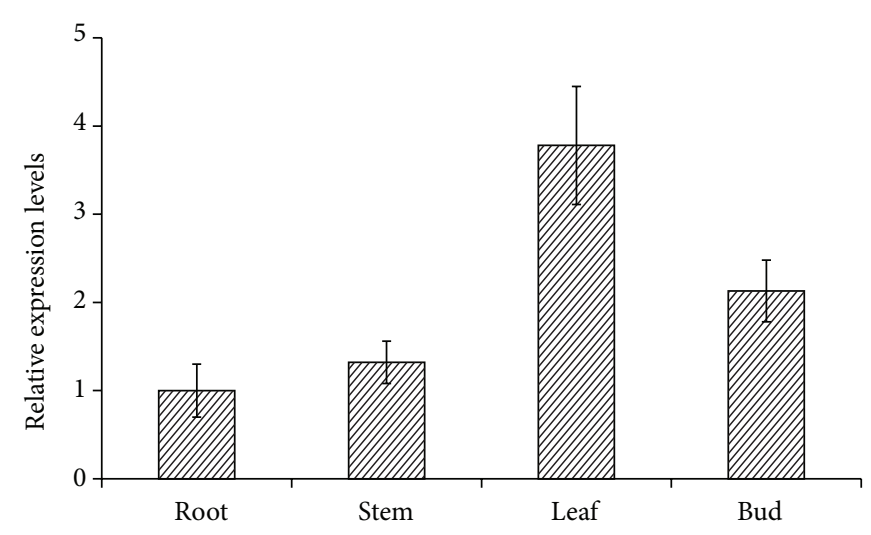

(b)

FIGURE 2: Expression profiling analysis of AaPP2C1. (a) qRT-PCR analysis of the expression pattern of AaPP2C1 under various environmental stress conditions. (b) AaPP2C1 expression in various tissues. The data represent the means \pm SD (standard deviation) of three repeated samples.

fused to GAD. In the case of yeast cells cotransformed with GAD-AaPP2C1m1 and GBD-AaPYL9 as well as the combination of $\mathrm{AD}-\mathrm{AaPP} 2 \mathrm{C} 1 \mathrm{~m} 2$ with BD-AaPYL9 did not activate transcription of the HIS3 and ADE2 reporter gene (Figure 3(b)). These results indicate that the two AaPP2C1 mutations, AaPP2C1m1 and $\mathrm{AaPP} 2 \mathrm{Clm} 2$, abolished the interaction with AaPYL9 in yeast two-hybrid; functional PP2C catalytic domain and the third phosphorylation site-like motif of $\mathrm{AaPP} 2 \mathrm{Cl}$ are required for the interaction of $\mathrm{AaPP} 2 \mathrm{Cl}$ and AaPYL9.

3.5. In Planta Interaction between AaPP2C1 and AaPYL9. Bimolecular fluorescence complementation (BiFC) assays were used to detect the interaction between AaPP2C1 and AaPYL9 in plant cell. To this end, AaPP2C1 was fused to the N-terminal (amino acid 1-174) of YFP protein $\left(\mathrm{YFP}^{\mathrm{N}}\right)$ in the pEG201YN vector, which generated an AaPP2C1-YFP ${ }^{\mathrm{N}}$ protein. AaPYL9 was fused to the C-terminal (amino acid 175-239) of YFP protein $\left(\mathrm{YFP}^{\mathrm{C}}\right.$ ) in the pEG202YC vector, which generated an AaPYL9-YFP ${ }^{C}$ protein. The corresponding constructs were coinfiltrated into leaf cells of tobacco; YFP fluorescence signal was observed in both of nucleus and cytosol (Figure 3(c)). As control, no fluorescence signal was observed when empty pEG201YN vector was coinfiltrated with pEG202YC-AaPYL9 or when pEG201YN-AaPP2C1 was coinfiltrated with empty pEG202YC vector (Figure 3(c)). Moreover, in accord with previous finding in the yeast two-hybrid assay, the AaPP2C1m1 and AaPP2C1m2 cannot interaction with AaPYL9 in the BiFC assay (Figure 3(d)).

3.6. Overexpression of AaPP2C1 Showed a Reduced Sensitivity to $A B A$ in Arabidopsis. The phenotype of AaPYL9overexpression in Arabidopsis was previously reported to be ABA hypersensitivity [26]. As AaPP2C1 interaction with
AaPYL9, we performed constitutively expression AaPP2C1 in Arabidopsis. As shown in Figure 4(a), the AaPP2C1overexpression lines exhibited higher frequency of seedlings with green cotyledons under $0.1 \mu \mathrm{MABA}$ or $0.3 \mu \mathrm{MABA}$ treatment compared with wild type. The percentage of seeds that germinated in the presence of indicated concentrations of ABA was also determined (Figure 4(b)). These assays reveal a reduced sensitivity to ABA of $A a P P 2 C 1$ overexpression lines. Moreover, this phenotype was apparent in root length assay, when seedlings were grown in MS medium with $5 \mu \mathrm{M}$ ABA or $10 \mu \mathrm{M}$ ABA for $7 \mathrm{~d}$. The roots of all the AaPP2C1-overexpression lines showed 50-95\% longer than wild type root (Figures $4(\mathrm{c})$ and $4(\mathrm{~d})$ ). These results suggest that $\mathrm{AaPP} 2 \mathrm{C} 1$ is a negative regulator of ABA signaling during seed germination and root elongation.

Further evidence of the function of $\mathrm{AaPP} 2 \mathrm{C} 1$ in $\mathrm{ABA}$ signaling was obtained through analyzing the expression of the ABA-responsive RD29A, RD29B, P5CS1, and RAB18 genes in the wild type and the AaPP2C1-overexpression lines by quantitative real-time PCR (qRT-PCR). Upon ABA treatment, the expression levels of RD29A, RD29B, P5CS1, and $R A B 18$ genes were significantly reduced in $\mathrm{AaPP} 2 \mathrm{C} 1$ overexpression lines compared with wild type (Figure 4(e)).

\section{Conclusions}

In this study, we functionally characterized AaPP2C1, a clade A PP2C, in Artemisia annua L. Previously, we reported an ABA receptor in Artemisia which act as a positive regulator in ABA signaling and play an important role in ABA signaling regulating artemisinin content. Yeast two-hybrid assay and $\mathrm{BiFC}$ analysis revealed a strong interaction between AaPYL9 and $\mathrm{AaPP} 2 \mathrm{C} 1$. Both the functional PP2C catalytic domain and the third phosphorylation site-like motif of AaPP2C1 appeared to be required for the interaction (Figure 3). 

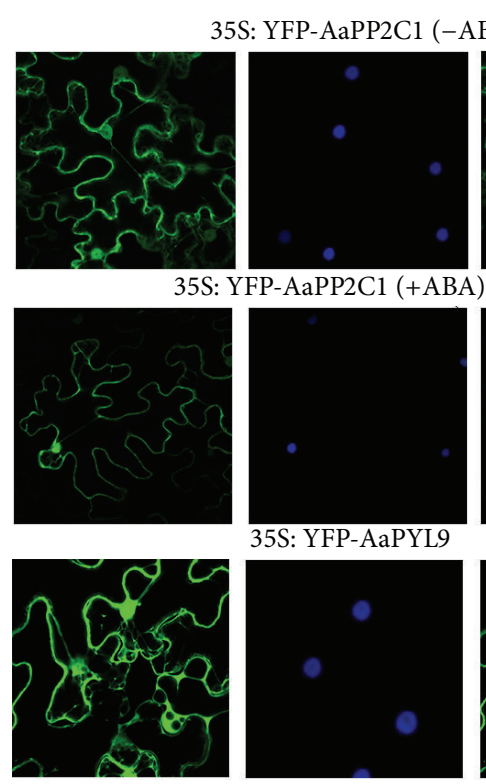

35S: YFP-AaPP2C1 (+ABA)
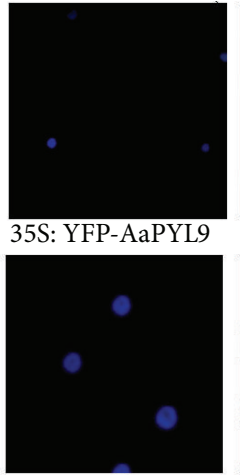

(a)

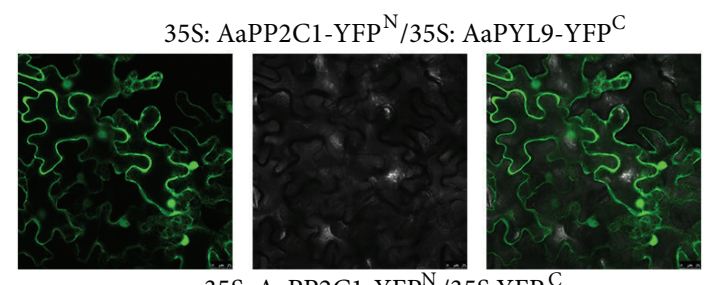

35S: AaPP2C1-YFP ${ }^{\mathrm{N}} / 35 \mathrm{~S}: \mathrm{YFP}^{\mathrm{C}}$
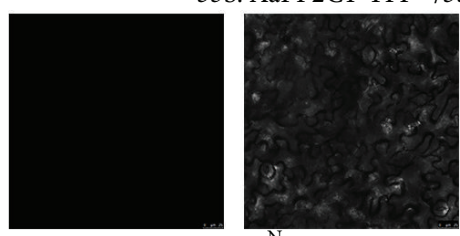

35S: YFP /35S: AaPYL9-YFP
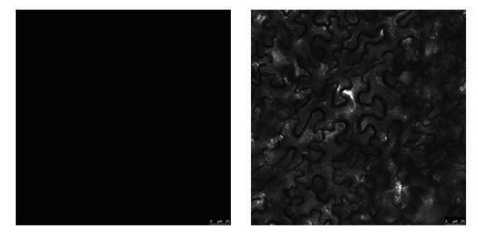

(c)
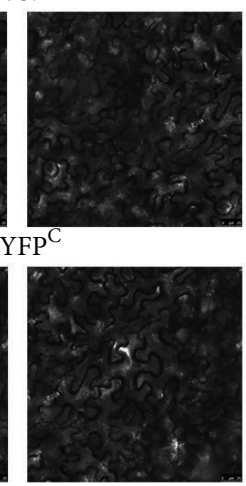

is

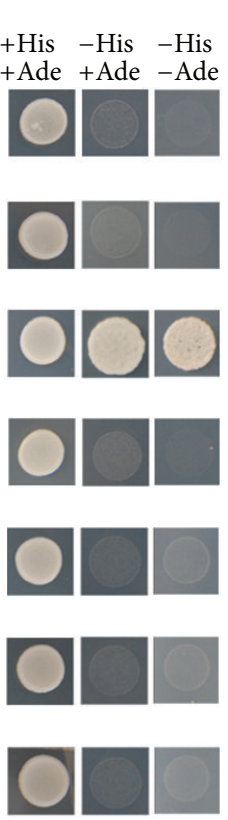

Bait

GBD

GBD-AaPYL9

GBD-AaPYL9

GBD-AaPYL9

GBD-AaPYL9

GBD-AaPYL9 m1

GBD-AaPYL9 m2

(b)
Prey

GAD-AaPP2C1

GAD

GAD-AaPP2C1

GAD-AaPP2C1 m1

GAD-AaPP2C1 m2

GAD-AaPP2C1

GAD-AaPP2C1
35S: AaPP2C1 m1-YFP ${ }^{\mathrm{C}} / 35 \mathrm{~S}:$ AaPYL9-YFP ${ }^{\mathrm{C}}$

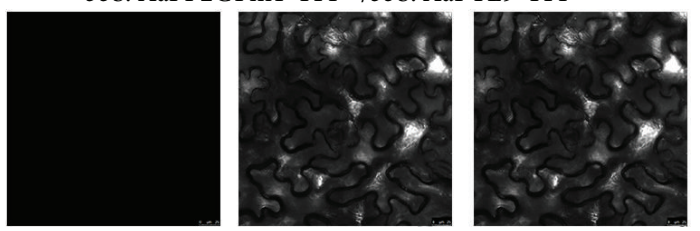

35S: AaPP2C1 m2-YFP $/ 35 S:$ AaPYL9-YFP ${ }^{\mathrm{C}}$
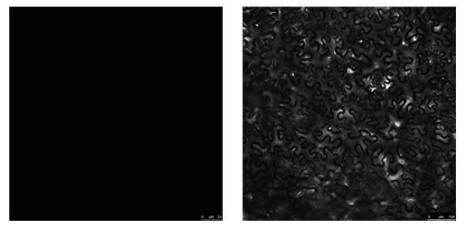

(d)

FIgURE 3: AaPP2C1 localizes at both cytosol and nucleus and interaction with AaPYL9. (a) Subcellular localization of AaPP2C1 and AaPYL9 in Agrobacterium-infiltrated tobacco leaves. Nucleus was visualized by DAPI staining. Left panel: YFP channel; middle panel: DAPI channel; right panel: merged picture. Treatment with $10 \mu \mathrm{M}$ ABA for $1 \mathrm{~h}$ did not change the subcellular localization of AaPP2C1. (b) The construct of genes fused $\mathrm{N}$ - and C-terminal to YFP, respectively (as indicated), were coinfiltrated into tobacco leaves. YFP signal was observed $48 \mathrm{~h}$ to $60 \mathrm{~h}$ after infiltrated. (c) The BiFC assay between two mutations of AaPP2C1 and AaPYL9. (d) Yeast two-hybrid assay confirming the interaction between $\mathrm{AaPP} 2 \mathrm{Cl}$ and AaPYL9. Bait indicates the protein fusion with Gal4 BD domain. Prey indicates the protein fusion with Gal4 AD domain. GBD is empty pGBKT7 vector, GAD is empty pGADT7 vector. (+His, +Ade) indicates Leu-Trp SD medium; (-His, +Ade) indicates Leu-Trp-His SD medium; (-His, -Ade) indicates Leu-Trp-His-Adedeficient SD medium. AaPYL9m1: substitution mutation P89S; AaPYL9m2: substitution mutation H116A. AaPP2C1m1: substitution mutation G199D; AaPP2C1m2: deletion mutation (four amino acid residues from 305 to 308: RGKE). 


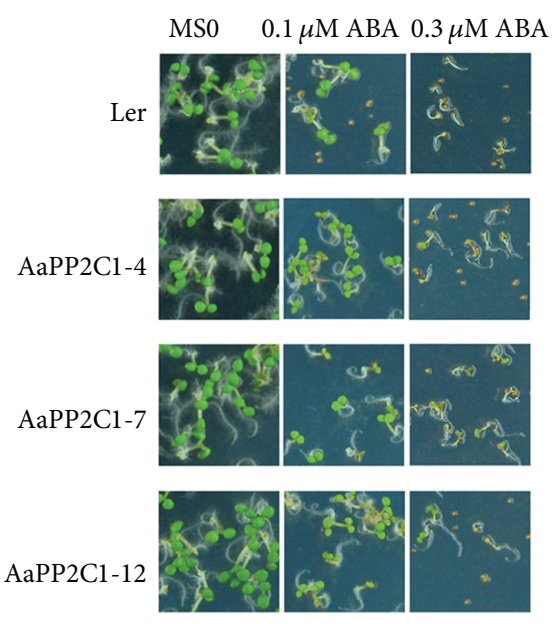

(a)

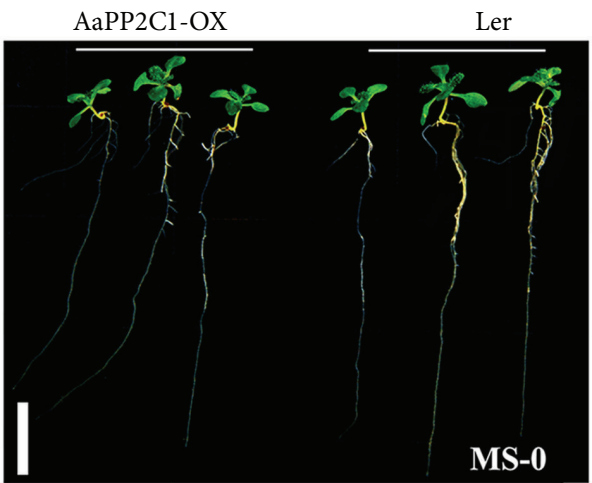

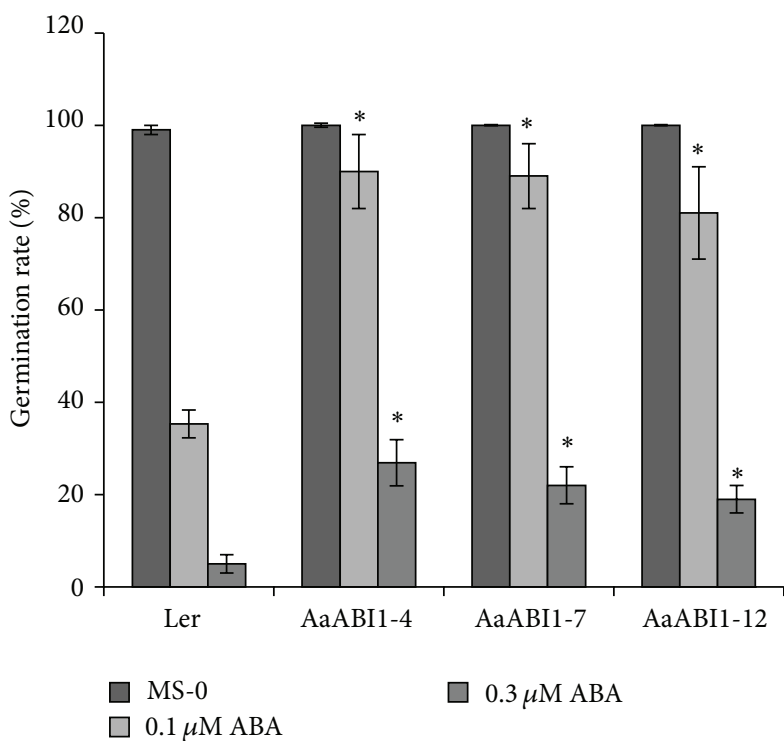

(b)

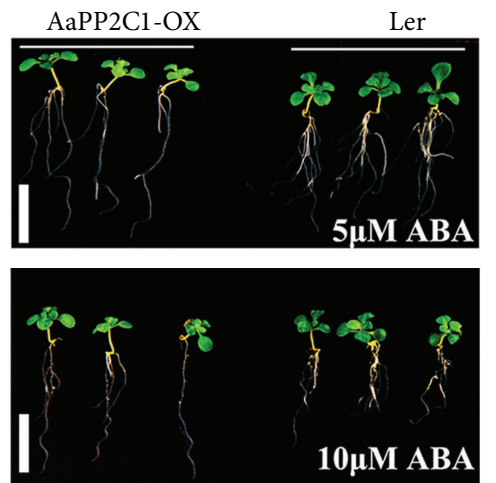

(c)

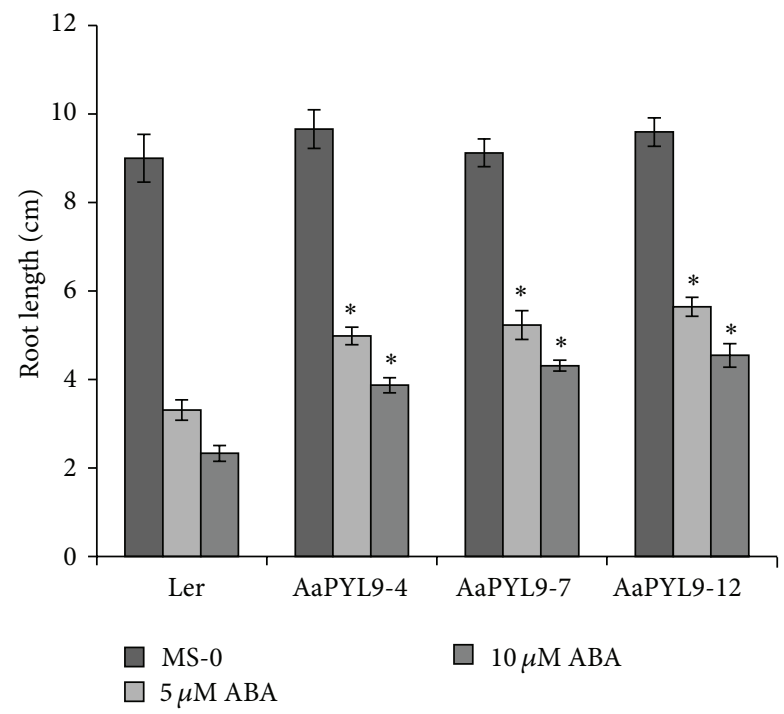

(d)

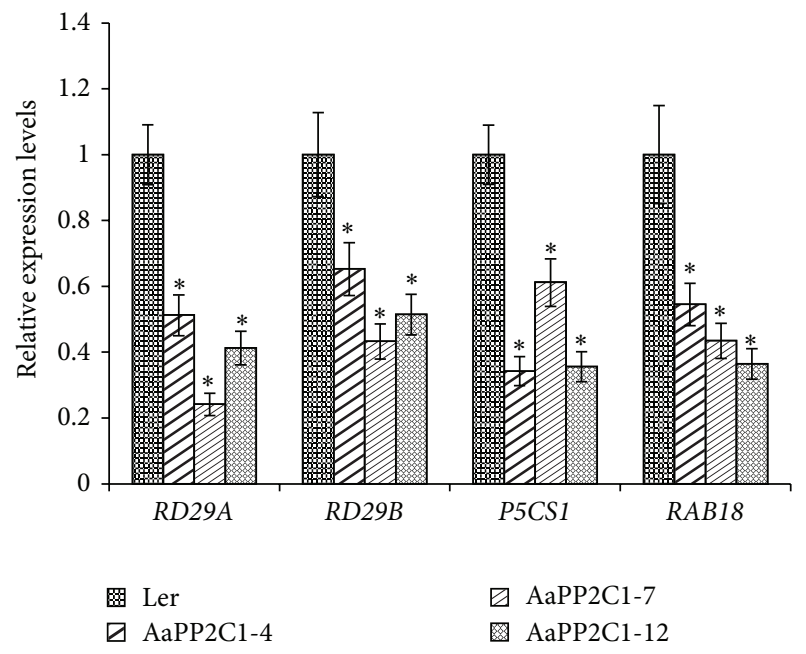

(e)

Figure 4: Comparative analysis of seed germination and root length between AaPP2C1-OX and wild type Arabidopsis. (a) Seed germination rate of AaPP2C1-OX Arabidopsis higher than wild type. (b) Statistics of seed germination rate. (c) The root length of AaPP2C1-OX Arabidopsis longer than wild type in the presence of the indicated ABA concentrations is shown. (d) Statistics of root length. (e) Reduced expression of ABA-inducible genes in AaPP2C1 overexpression Arabidopsis. The mRNA levels of the indicated genes were determined by Q-PCR analysis using total RNAs isolated from $10 \mu \mathrm{M}$ ABA-treated plants for $3 \mathrm{~h}$. SD (standard deviation) indicates three independent tests. ${ }^{*} P<0.01, t$-test. 
Overexpression of $\mathrm{AaPP} 2 \mathrm{Cl}$ in Arabidopsis reduced ABA sensitively in seed germination and root elongation revealed that $\mathrm{AaPP} 2 \mathrm{Cl}$ is a negative regulator of $\mathrm{ABA}$ signaling (Figure 4). Taken together, our data suggests that AaPP2C1 is a functional PP2C in Artemisia, showing that the core regulatory mechanism of early ABA signaling in Artemisia is conserved with Arabidopsis. This is particularly important for investigating the mechanism ABA signaling regulation secondary metabolism; AaPYL9 especially has been reported to be involved in regulating artemisinin biosynthesis.

\section{Conflict of Interests}

The authors declare that there is no conflict of interests regarding the publication of this paper.

\section{Acknowledgments}

The authors are very grateful to Professor Hongquan Yang (Shanghai Jiao Tong University) for providing help in experiment. They appreciate Dr. Fei Zhang's (Oklahoma State University) fruitful discussions and kind suggestions. This work was funded by China National High-Tech 863 Program (2011AA100605) and Shanghai Leading Academic Discipline Project (Horticulture).

\section{References}

[1] S. R. Cutler, P. L. Rodriguez, R. R. Finkelstein, and S. R. Abrams, "Abscisic acid: emergence of a core signaling network," Annual Review of Plant Biology, vol. 61, pp. 651-679, 2010.

[2] J.-K. Zhu, "Salt and drought stress signal transduction in plants," Annual Review of Plant Biology, vol. 53, pp. 247-273, 2002.

[3] T. Yoshida, Y. Fujita, H. Sayama et al., "AREB1, AREB2, and $\mathrm{ABF} 3$ are master transcription factors that cooperatively regulate ABRE-dependent ABA signaling involved in drought stress tolerance and require ABA for full activation," Plant Journal, vol. 61, no. 4, pp. 672-685, 2010.

[4] Y. Ma, I. Szostkiewicz, A. Korte et al., "Regulators of PP2C phosphatase activity function as abscisic acid sensors," Science, vol. 324, no. 5930, pp. 1064-1068, 2009.

[5] N. Nishimura, A. Sarkeshik, K. Nito et al., "PYR/PYL/RCAR family members are major in-vivo ABI1 protein phosphatase 2C-interacting proteins in Arabidopsis," Plant Journal, vol. 61, no. 2, pp. 290-299, 2010.

[6] F. F. Soon, L. M. Ng, X. E. Zhou et al., "Molecular mimicry regulates ABA signaling by SnRK2 kinases and PP2C phosphatases," Science, vol. 335, no. 6064, pp. 85-88, 2012.

[7] B. Brandt, D. E. Brodsky, S. Xue et al., "Reconstitution of abscisic acid activation of SLAC1 anion channel by CPK6 and OST1 kinases and branched ABI1 PP2C phosphatase action," Proceedings of the National Academy of Sciences of the United States of America, vol. 109, no. 26, pp. 10593-10598, 2012.

[8] T. Umezawa, N. Sugiyama, M. Mizoguchi et al., “Type 2C protein phosphatases directly regulate abscisic acid-activated protein kinases in Arabidopsis," Proceedings of the National Academy of Sciences of the United States of America, vol. 106, no. 41, pp. 17588-17593, 2009.
[9] T. Xue, D. Wang, S. Zhang et al., "Genome-wide and expression analysis of protein phosphatase $2 \mathrm{C}$ in rice and Arabidopsis," BMC Genomics, vol. 9, article 550, 2008.

[10] R. Antoni, M. Gonzalez-Guzman, L. Rodriguez, A. Rodrigues, G. A. Pizzio, and P. L. Rodriguez, "Selective inhibition of clade A phosphatases type 2C by PYR/PYL/RCAR abscisic acid receptors," Plant Physiology, vol. 158, no. 2, pp. 970-980, 2012.

[11] G. B. Bhaskara, T. T. Nguyen, and P. E. Verslues, "Unique drought resistance functions of the highly ABA-induced clade a protein phosphatase 2Cs," Plant Physiology, vol. 160, no. 1, pp. 379-395, 2012.

[12] R. Yoshida, T. Umezawa, T. Mizoguchi, S. Takahashi, F. Takahashi, and K. Shinozaki, "The regulatory domain of SRK2E/OST1/SnRK2.6 interacts with ABI1 and integrates abscisic acid (ABA) and osmotic stress signals controlling stomatal closure in Arabidopsis," Journal of Biological Chemistry, vol. 281, no. 8, pp. 5310-5318, 2006.

[13] S. Merlot, F. Gosti, D. Guerrier, A. Vavasseur, and J. Giraudat, "The ABI1 and ABI2 protein phosphatases $2 \mathrm{C}$ act in a negative feedback regulatory loop of the abscisic acid signalling pathway," Plant Journal, vol. 25, no. 3, pp. 295-303, 2001.

[14] H.-F. Jia, D. Lu, J.-H. Sun et al., "Type 2C protein phosphatase ABI1 is a negative regulator of strawberry fruit ripening," Journal of Experimental Botany, vol. 64, no. 6, pp. 1677-1687, 2013.

[15] H. Kim, H. Hwang, J. W. Hong et al., "A rice orthologue of the ABA receptor, OsPYL/RCAR5, is a positive regulator of the ABA signal transduction pathway in seed germination and early seedling growth," Journal of Experimental Botany, vol. 63, no. 2, pp. 1013-1024, 2012.

[16] O. Lorenzo, D. Rodríguez, G. Nicolás, P. L. Rodríguez, and C. Nicolás, "A new protein phosphatase 2C (FsPP2C1) induced by abscisic acid is specifically expressed in dormant beechnut seeds," Plant Physiology, vol. 125, no. 4, pp. 1949-1956, 2001.

[17] X. Saavedra, A. Modrego, D. Rodríguez et al., "The nuclear interactor PYL8/RCAR3 of Fagus sylvatica FsPP2C1 is a positive regulator of abscisic acid signaling in seeds and stress," Plant Physiology, vol. 152, no. 1, pp. 133-150, 2010.

[18] L. Liu, X. Hu, J. Song, X. Zong, and D. Li, “Over-expression of a Zea mays L. protein phosphatase $2 \mathrm{C}$ gene (ZmPP2C) in Arabidopsis thaliana decreases tolerance to salt and drought," Journal of Plant Physiology, vol. 166, no. 5, pp. 531-542, 2009.

[19] K. Komatsu, Y. Nishikawa, T. Ohtsuka et al., "Functional analyses of the ABI1-related protein phosphatase type $2 \mathrm{C}$ reveal evolutionarily conserved regulation of abscisic acid signaling between Arabidopsis and the moss Physcomitrella patens," Plant Molecular Biology, vol. 70, no. 3, pp. 327-340, 2009.

[20] L. A. Graham, K. Besser, S. Blumer et al., "The genetic map of Artemisia annua L. identifies loci affecting yield of the antimalarial drug artemisinin," Science, vol. 327, no. 5963, pp. 328-331, 2010.

[21] W. Wang, Y. Wang, Q. Zhang, Y. Qi, and D. Guo, "Global characterization of Artemisia annua glandular trichome transcriptome using 454 pyrosequencing," BMC Genomics, vol. 10, article 465, 2009.

[22] M. R. Romero, M. A. Serrano, M. Vallejo, T. Efferth, M. Alvarez, and J. J. G. Marin, "Antiviral effect of artemisinin from Artemisia annua against a model member of the Flaviviridae family, the bovine viral diarrhoea virus (BVDV)," Planta Medica, vol. 72, no. 13, pp. 1169-1174, 2006. 
[23] T. Efferth, "Molecular pharmacology and pharmacogenomics of artemisinin and its derivatives in cancer cells," Current Drug Targets, vol. 7, no. 4, pp. 407-421, 2006.

[24] J. Utzinger, S.-H. Xiao, M. Tanner, and J. Keiser, "Artemisinins for schistosomiasis and beyond," Current Opinion in Investigational Drugs, vol. 8, no. 2, pp. 105-116, 2007.

[25] F. Jing, L. Zhang, M. Li et al., "Abscisic acid (ABA) treatment increases artemisinin content in Artemisia annua by enhancing the expression of genes in artemisinin biosynthetic pathway," Biologia, vol. 64, no. 2, pp. 319-323, 2009.

[26] F. Zhang, X. Lu, Z. Lv et al., "Overexpression of the artemisia orthologue of ABA receptor, AaPYL9, enhances ABA sensitivity and improves artemisinin content in Artemisia annua L," PLoS ONE, vol. 8, no. 2, Article ID e56697, 2013.

[27] K. W. Earley, J. R. Haag, O. Pontes et al., "Gateway-compatible vectors for plant functional genomics and proteomics," Plant Journal, vol. 45, no. 4, pp. 616-629, 2006.

[28] Q. Lu, X. Tang, G. Tian et al., "Arabidopsis homolog of the yeast TREX-2 mRNA export complex: components and anchoring nucleoporin," Plant Journal, vol. 61, no. 2, pp. 259-270, 2010.

[29] S.-Y. Park, P. Fung, N. Nishimura et al., "Abscisic acid inhibits type $2 \mathrm{C}$ protein phosphatases via the PYR/PYL family of START proteins," Science, vol. 324, no. 5930, pp. 1068-1071, 2009.

[30] A. Schweighofer, H. Hirt, and I. Meskiene, "Plant PP2C phosphatases: Emerging functions in stress signaling," Trends in Plant Science, vol. 9, no. 5, pp. 236-243, 2004.

[31] H.-L. Sun, X.-J. Wang, W.-H. Ding et al., "Identification of an important site for function of the type $2 \mathrm{C}$ protein phosphatase ABI2 in abscisic acid signalling in Arabidopsis," Journal of Experimental Botany, vol. 62, no. 15, pp. 5713-5725, 2011. 

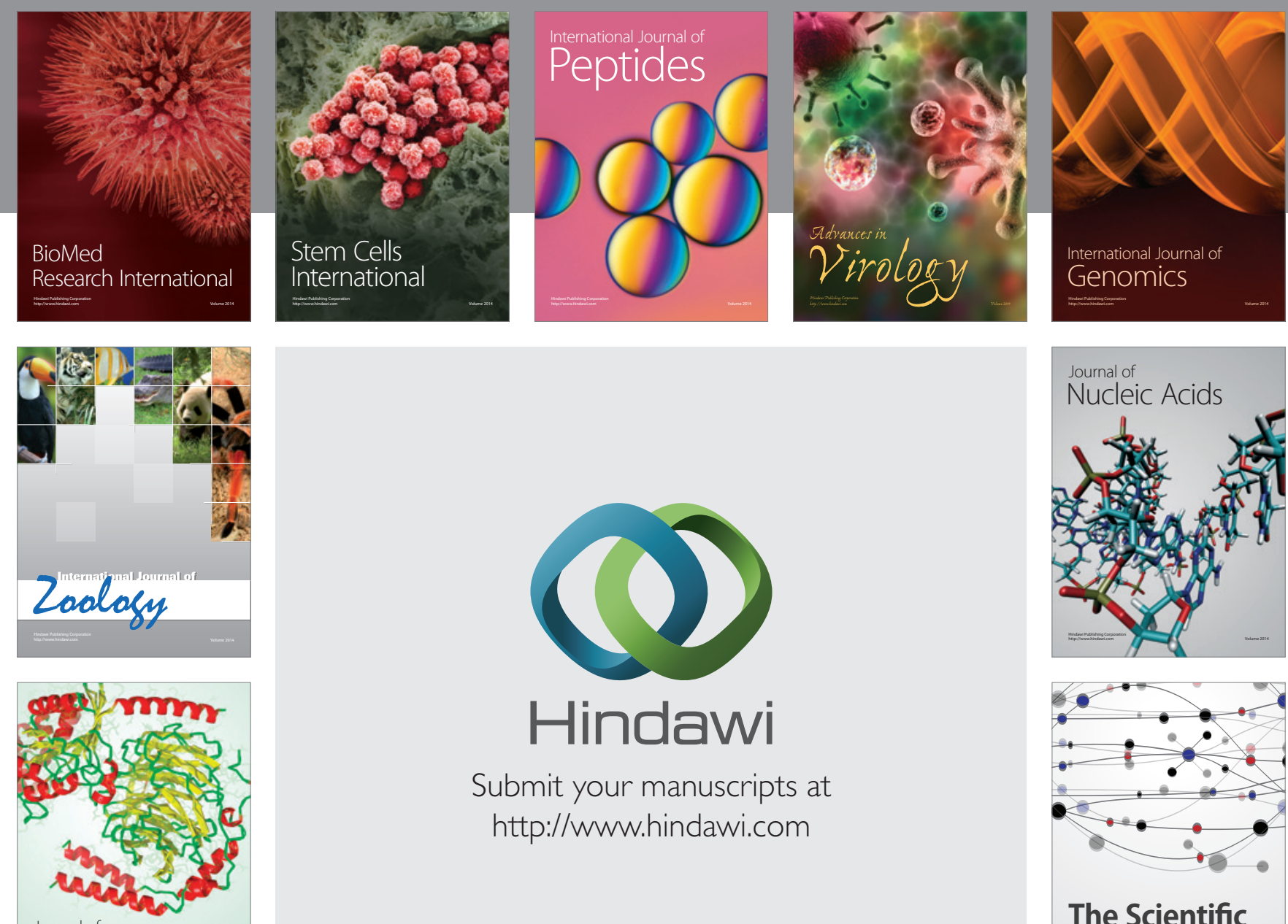

Submit your manuscripts at

http://www.hindawi.com

Journal of
Signal Transduction
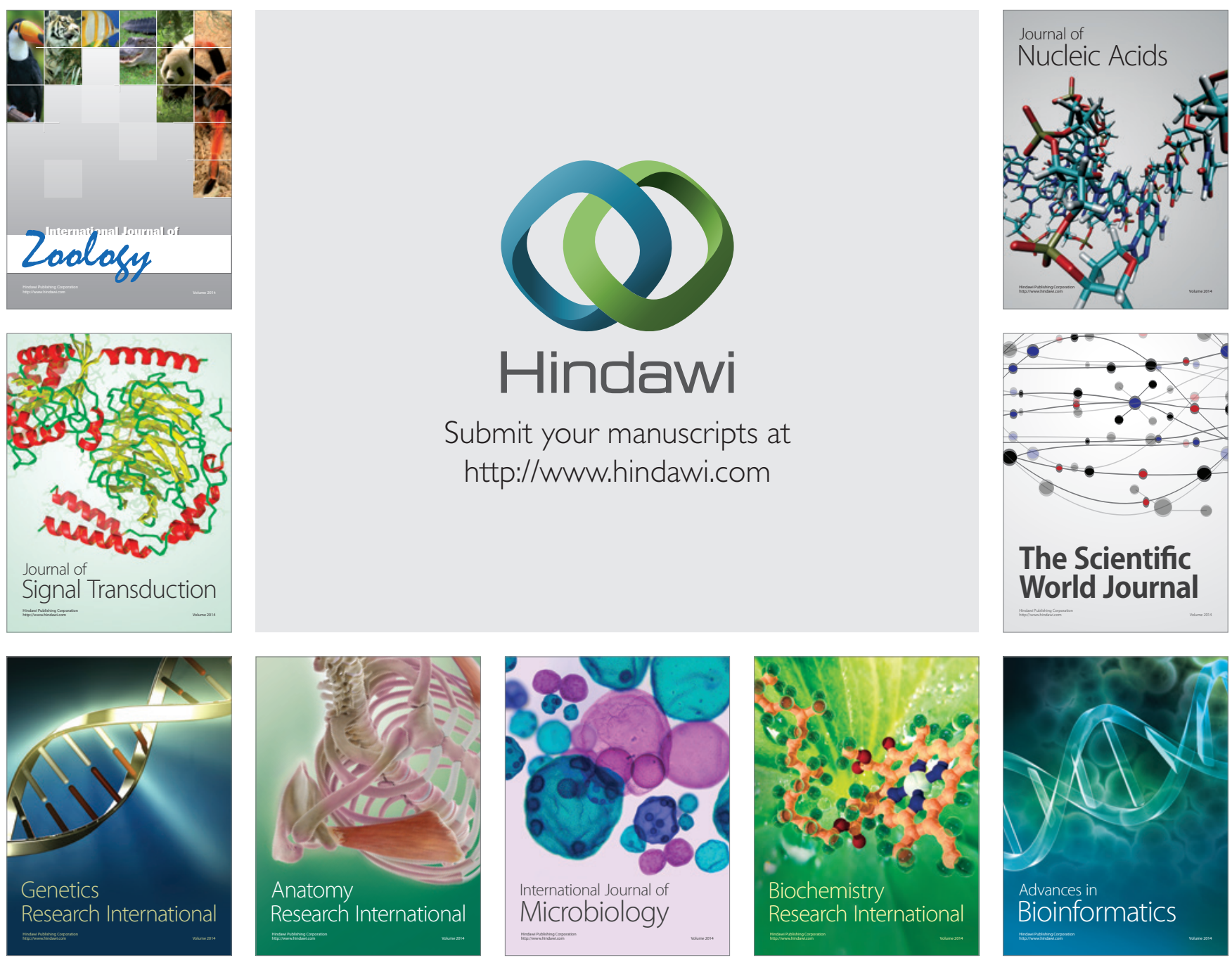

The Scientific World Journal
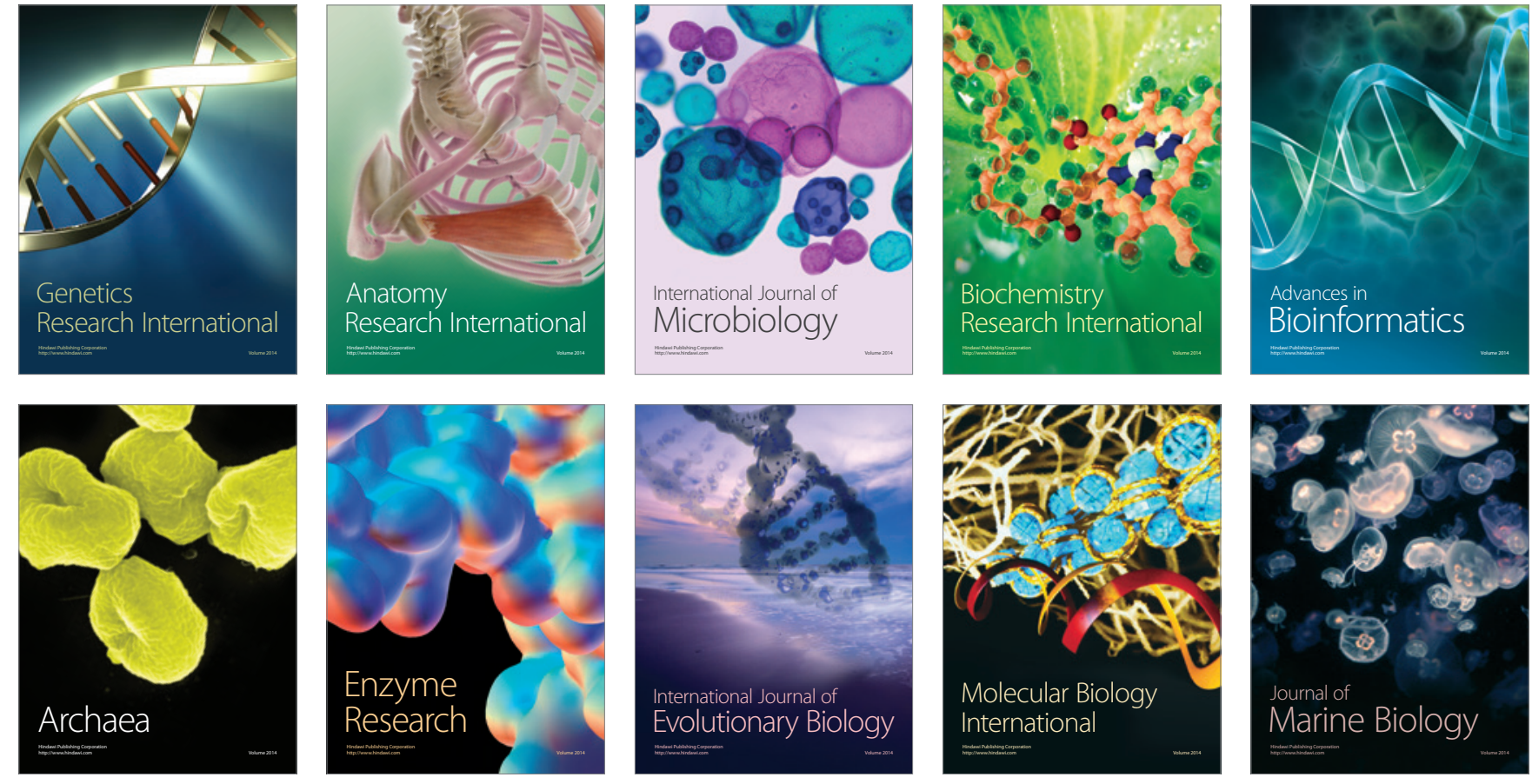